\title{
Ceramic high-rate timing RPCs
}

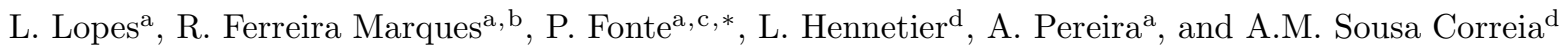

a Laboratório de Instrumentação e Física Experimental de Partículas, 3004-516 Coimbra, Portugal

${ }^{\text {b}}$ Departamento de Física, Faculdade de Ciências e Tecnologia da Universidade de Coimbra, 3004-516

Coimbra, Portugal

${ }^{\mathrm{c}}$ Instituto Superior de Engenharia de Coimbra, Quinta da Nora, 3030-199 Coimbra, Portugal

${ }^{\mathrm{d}}$ Centro Tecnológico da Ceramica e do Vidro, Rua Coronel Veiga Simão, 3020-053 Coimbra

Following some previous work, we report here considerable improvements on the counting rate capability of timing RPCs by the use of ceramic electrodes with a resistivity of $10^{9} \Omega \cdot \mathrm{cm}$. The X-ray sensitivity of the detector depends linearly on the counting rate with a slope of $9 \%$ per $100 \mathrm{kHz} / \mathrm{cm}^{2}$, free from charge depletion effects, while keeping a timing accuracy, measured with $511 \mathrm{keV}$ synchronous photon pairs, around $90 \mathrm{ps} \sigma$ up to $75 \mathrm{kHz} / \mathrm{cm}^{2}$.

(C) 2001 Elsevier Science. All rights reserved

Keywords: RPC; TOF; High-rate; Ceramic electrode

\section{Introduction}

Timing resistive plate chambers [1] are planar gaseous detectors made with a combination of metallic and resistive electrodes, which deliver a time resolution around sigma $(\sigma) 50 \mathrm{ps}$ and efficiency up to $99 \%$ for MIPs.

The counting rate capability of these detectors is mainly determined by the resistivity of the electrodes, which are typically made of glass with volume resistivity between $10^{12}$ and $10^{13} \Omega \cdot \mathrm{cm}$. This limits the maximum counting rate to around $2 \mathrm{kHz} / \mathrm{cm}^{2}$ [2], which may be a strong drawback for some applications, as, for instance, the proposed CBM [3] heavy ion experiment.

In this work we report on the use of a ceramic material with a measured resistivity of $10^{9} \Omega \cdot \mathrm{cm}$ and free from charge depletion effects [4].

\section{Materials for high-rate timing RPCs}

The effective voltage applied on the gas gap of an RPC is given by

$$
V_{e f f}=V_{a p}-I R=V_{a p}-\left(\frac{I}{A}\right) \rho l
$$

*Corresponding author. Tel.: +351-239833465; fax: +351-239822358; e-mail: fonte@coimbra.lip.pt. where $V_{\text {eff }}$ is the effective voltage applied across the gap, $V_{a p}$ is the externally applied voltage, $I$ is the counting current drawn by the detector in a reference area $A, R$ is the electrical resistance seen by this current, $\rho$ is the DC bulk resistivity of the electrode resistive material, which may be a function of time, integrated charge and voltage drop across it, and lis the total thickness of resistive material crossed by the current $I$. It is clear that for a given current density $(I / A)$ the relevant electrode characteristic is $\rho l$.

The stringent planarity requirements of timing RPCs (around $10 \%$ of the gap width [5] require the use of electrodes that are either produced quite flat, like industrial flat glass, or are homogeneous and hard enough to be lapped. This excludes inhomogeneous materials, as the very commonly used bakelite.

Naturally, one finds in the literature an immense variety of in-principle suitable materials, in the general categories of glasses, ceramics and polymers. However, the path between a given promising material and a mass-producible detector is a long and risky one, being advisable to restrain our choice of materials to the commercially available industrial products. 
Unfortunately, most of these materials have not been characterized for the conductivity type. Many show a dependence of the apparent resistivity with the integrated charge that results from the opposing electric field created by the transport and accumulation of the ionic charge carriers on the material surfaces, an effect commonly known as the "charge depletion" or "charging up" effect. This is typically the case of polymers, such as low resistivity baquelite [6] or antistatic Ensital [7].

It has been also found that the resistivity of sodalime and borosilicate glasses, although free from the charge depletion effect, is exponentially dependent on the temperature by a remarkably constant ratio of one order of magnitude per each $25^{\circ} \mathrm{C}$ of temperature increase [8], [9]. This provides a rather practical way of increasing the rate capability of glass RPCs by the sole means of a moderate warming of the detectors.

In Table 1 we list a few materials that may be of interest for high-rate timing RPCs. The first line corresponds to a common sodalime glass, which at $20^{\circ} \mathrm{C}$ allows maximum counting rates close to $200 \mathrm{~Hz} / \mathrm{cm}^{2}$ when irradiated with photons and $600 \mathrm{~Hz} / \mathrm{cm}^{2}$ for charged particles [9]. A comparison with the last line allows us to expect maximum counting rates four orders of magnitude larger when using the proposed ceramic material.

\begin{tabular}{cccc}
$\begin{array}{c}\text { Manufacturer - } \\
\text { Type }\end{array}$ & $\begin{array}{c}\text { Thickness } \\
(\mathrm{mm})\end{array}$ & $\begin{array}{c}\rho_{20} l \\
\left(10^{12}\right. \\
\left.\Omega \mathrm{cm}^{2}\right)\end{array}$ & $\begin{array}{c}\Delta T \\
\left({ }^{\circ} \mathrm{C}\right)\end{array}$ \\
\hline Glaverbel - clear & 1.9 & 2.00 & 24.3 \\
idem & 0.4 & 0.281 & 23.5 \\
SCHOTT - D263T & $0.3^{1}$ & 186 & 23.9 \\
Morgan ceramics & 2.0 & $2.14 \times$ & - \\
-AL940CD [4] & & $10^{-4}$ &
\end{tabular}

Table 1

List of a few materials of interest for timing RPCs. The first two lines correspond to a common sodalime ${ }^{2}$ glass, the third line is a borosilicate $^{3}$ glass and the last line concerns the ceramic material studied in this work. The parameter $\Delta T$ is the temperature increase that will result in a resistivity decrease by one order of magnitude [9].
Finally, it is worth mentioning that a low dielectric constant material is required, otherwise the large capacity created will allow the development of inconveniently large discharges. This excludes potentially very interesting materials like bulk SiC, but layers of such class of materials may be deposited on dielectric substrates with good results [10]. This approach may, however, be limited to rather small area detectors or to low rates, as the effective applied voltage will be inhomogeneous along the detector and the avalanche average time delay has a slope of approximately $1 \mathrm{ps} / \mathrm{V}[11]$.

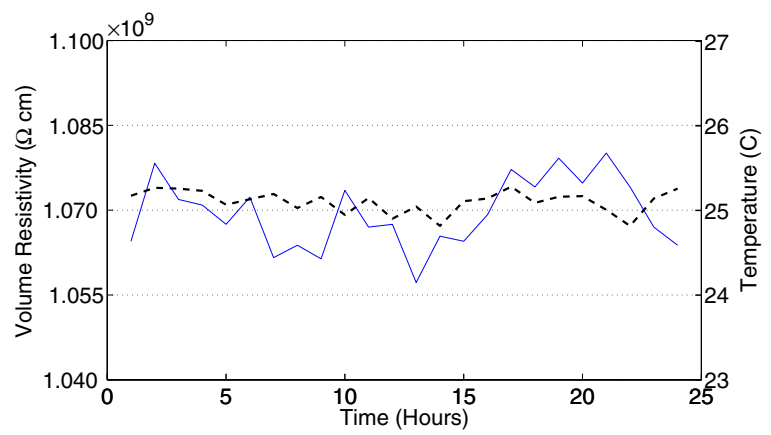

Figure 1. Volume resistivity evolution in time (full line), at controlled temperature (dashed line), while applying a $350 \mathrm{~V}$ potential. No significant variations in volume resistivity are observed, suggesting that this material is free from the charge depletion effect.

\section{Detector and experimental setup}

\subsection{The material}

The ceramic material is commercially available as a "controlled resistivity" alumina [4] with a measured resistivity of $1.07 \times 10^{9} \Omega \cdot \mathrm{cm}$, as it

\footnotetext{
${ }^{1}$ Available down to $0.1 \mathrm{~mm}$ thickness.

${ }^{2} \mathrm{~A}$ typical sodalime glass is composed of $\mathrm{SiO}_{2}$ (silica: 70$75 \%$ ), $\mathrm{Na}_{2} \mathrm{CO}_{3}$ (soda: 12-16\%) and $\mathrm{CaO}$ (lime: 5-15\%), plus small amounts of other materials to provide particular properties such as colour

${ }^{3}$ Borosilicate glasses are made mainly of silica (70-80\%) and boric oxide (7-13\%) with smaller amounts of the alkalis (sodium and potassium oxides) and aluminium oxide.
} 
may be seen in Fig. 1. The material was subjected to an external potential of $350 \mathrm{~V}$ at room temperature for a rather long time, mimicking the working conditions expected in a high-rate RPC. No systematic variations of the volume resistivity were observed, suggesting that the material is free from charge depletion effects.

Unfortunately the material is not fully uniform, including embedded low resistivity paths that emerge at discrete points of the surface. While in operation at large gas gains such paths may sustain permanent discharges, a phenomenon to be absolutely avoided. It is not know at this moment whether these paths are an unavoidable feature of this type of material or the situation may be improved by some change on the manufacturing process.

The problem was solved by segmenting the metallic electrode that polarises the back face of the ceramic electrode in a number of strips and grounding each strip through a resistor that assures a minimum electrical resistance in series with any discharge.

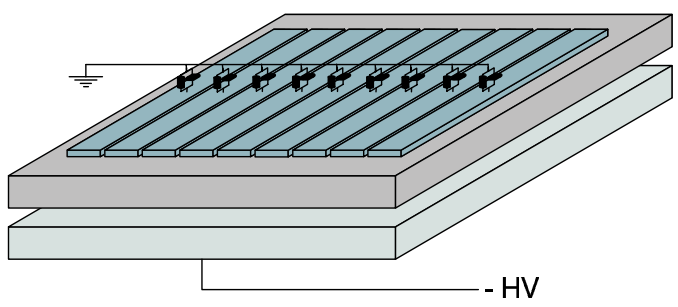

Figure 2. Schematic representation of the detector. The effective area of the resistive electrode was segmented in $1 \mathrm{~cm}^{2}$ The strips and each strip grounded trough a parallel RC circuit.

\subsection{Detector construction}

The detector, with an active area of $9 \mathrm{~cm}^{2}$, was composed by a $5 \mathrm{~mm}$ thick stainless steel cathode and a $2.5 \mathrm{~mm}$ thick ceramic anode, defining a single $0.3 \mathrm{~mm}$ wide gas gap (Fig. 2). Both electrodes had the active surfaces lapped and polished and their smoothed edges were externally glued to support columns [12]. As mentioned above, the anode back face was segmented into strips of
$1 \mathrm{~cm}^{2}$ effective area made from conductive silverloaded epoxy glue. Each strip is grounded via a parallel $\mathrm{RC}$ circuit, with $\mathrm{R}=470 \mathrm{M} \Omega$ and $\mathrm{C}=1 \mathrm{nF}$, that assured the required minimum series resistance while allowing a low impedance path for the signals.

\subsection{Experimental setup}

The experimental setup was already used in previous studies and a detailed description may be found in [13]. It follows essentially the same principle as CERN's GIF facility, the role of the gamma source being played by an X-ray tube and the muon test beam replaced by a weak ${ }^{22} \mathrm{Na}$ positron source emitting synchronous $511 \mathrm{keV}$ photon pairs.

The working gas mixture was constituted by $90 \%$ tetrafluorethane $\left(\mathrm{C}_{2} \mathrm{H}_{2} \mathrm{~F}_{4}\right)$ and $10 \%$ sulphur hexafluoride $\left(\mathrm{SF}_{6}\right)$, without any isobutane [7],[13].

\section{Results}

\subsection{Current and counting rate behaviour}

In Fig. 3 a) we show the counting current versus applied voltage for a set of X-ray anode currents, proportional to the primary particle flux. The almost linear dependence observed is known to be due to space charge effects in the progressing avalanche [6]. For the larger currents, around $850 \mathrm{nA} / \mathrm{cm}^{2}\left(240 \mathrm{kHz} / \mathrm{cm}^{2}\right.$, mean avalanche charge $3.4 \mathrm{pC}$ ), the voltage drop across the ceramic electrode and the series resistor should be close to $600 \mathrm{~V}$.

Figure $3 \mathrm{~b}$ ) shows the counting rate versus applied voltage for the same set of X-ray anode currents. A reasonably flat counting plateau is reached for X-ray anode currents up to $14 \mathrm{~mA}$, corresponding to chamber counting rates of more than $100 \mathrm{kHz} / \mathrm{cm}^{2}$.

At the highest applied voltage the background counting rate was less than $10 \mathrm{~Hz} / \mathrm{cm}^{2}$, and the corresponding background current less than $0.1 \mathrm{nA} / \mathrm{cm}^{2}$.

The counting rate density as a function of the X-ray tube current for a set of applied voltages is shown in Fig. 4 a). For each voltage the data is well described by a quadratic polynomial. The 


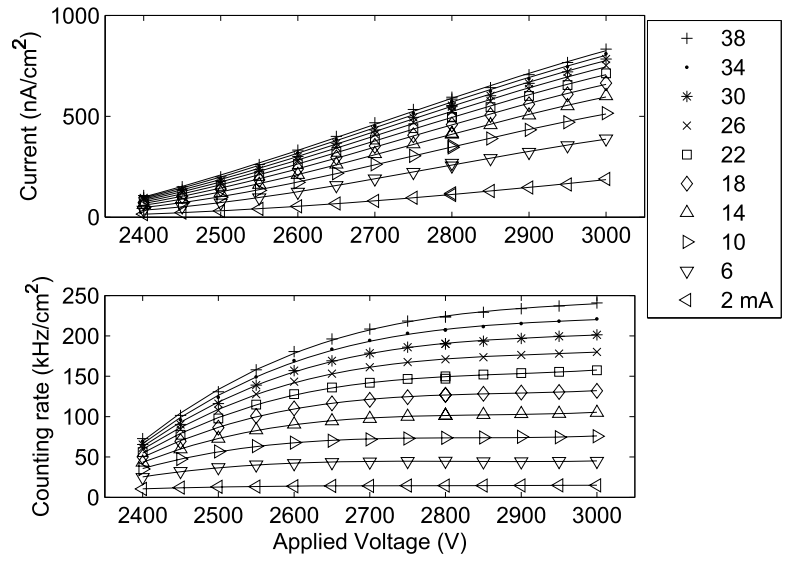

Figure 3. a) Operation current density as a function of the applied voltage for a set of X-ray anode currents (proportional to the incident particle flux). b) Counting rate density as function of the applied voltage. Lines were added to guide the eye.

relative sensitivity drop that may be attributed to the increasing counting rate (Fig. 4 b)) is estimated by normalizing the data points to the linear part of the fitting polynomial (the "incident" counting rate, also represented in a)). A linear fit to the set of the normalized points shows a slope of $9 \%$ relative sensitivity drop per $100 \mathrm{kHz} / \mathrm{cm}^{2}$.

It should be noted that when timing RPCs are irradiated with $\mathrm{X}$ - or gamma rays the avalanche charge distribution shows an exponential shape, instead of the shallow distribution observed with a charged beam [15]. Therefore, the efficiency is likely to be more sensitive to the operational parameters than what would be observed in a particle beam.

This applies also indirectly to the timing accuracy measurements, as the needed correction of time as a function of the avalanche charge is more difficult for an exponential charge distribution than for a shallow one.

\subsection{Time resolution}

The time resolution of the high rate chamber was estimated by quadratic subtraction of the resolution of the reference chamber from the mea- sured time difference spectrum. A detailed description may be found in [13].

The results are shown in Fig. 5. Within the statistical fluctuations of the measurement (vertical spread of the points), the time resolution remains essentially unchanged around $90 \mathrm{ps} \sigma$, between 1 and $75 \mathrm{kHz} / \mathrm{cm}^{2}$. Beyond this point the measurement becomes impossible owing to the predominance of random coincidences (see [13]).
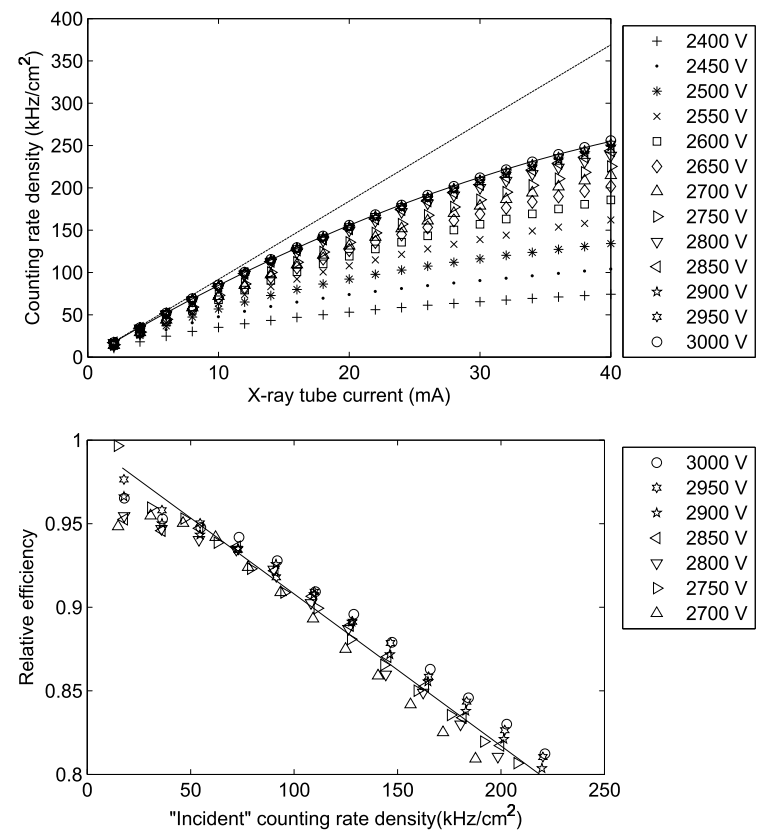

Figure 4. a) Counting rate density as a function of the X-ray tube current for a set of applied voltages. A fitting quadratic polynomial reproduces well the data (one example shown). b) The data points are normalized to the linear part of the fitting polynomial (the "incident" counting rate, also represented in a)), yielding the drop in relative efficiency that may be attributed to the counting rate. A general fit to these points shows a slope of $9 \%$ relative efficiency drop per $100 \mathrm{kHz} / \mathrm{cm}^{2}$.

It should be noted that the timing performance of timing RPCs in particle beams and with $511 \mathrm{keV}$ photons has been compared and it was found that with photons the resolution is systematically worse. 


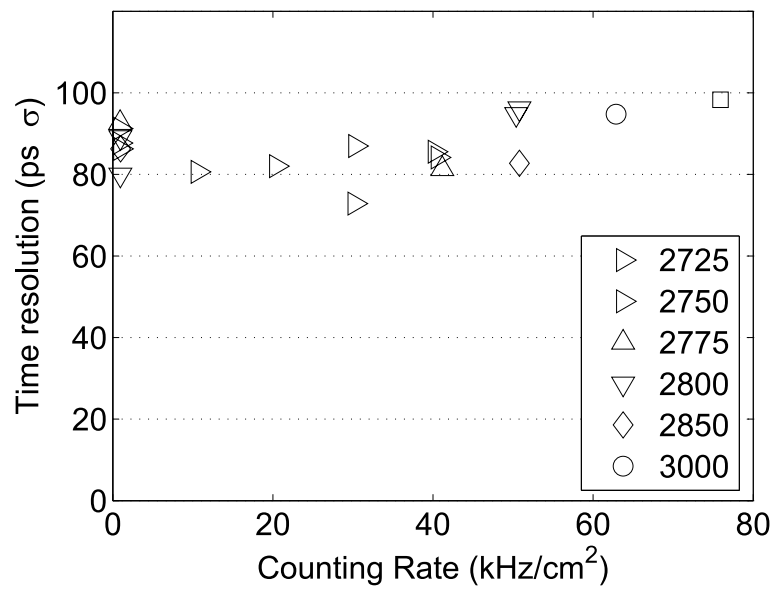

Figure 5. Time resolution as a function of the counting rate. Within the statistical fluctuations of the measurement, the time resolution remains essentially unchanged, around 90 ps $\sigma$, between 1 and $75 \mathrm{kHz} / \mathrm{cm}^{2}$. The applied voltage was adjusted as the counting rate increased in order to compensate for voltage change across the ceramic.

\section{Conclusion}

The present results establish the practical feasibility of accurate timing measurements with $\mathrm{RPCs}$ at rates up to $75 \mathrm{kHz} / \mathrm{cm}^{2}$, while keeping a time resolution below $100 \mathrm{ps} \sigma$ for $511 \mathrm{keV}$ gamma rays.

A linear rate-induced X-ray sensitivity drop of $9 \%$ per $100 \mathrm{kHz} / \mathrm{cm}^{2}$ was also observed. Likely, this is a more severe effect than what would be observed in a charged beam.

These results extend the field of application of timing RPCs to situations, as in CBM [3], where accurate timing over large areas at counting rates of tens of $\mathrm{kHz} / \mathrm{cm}^{2}$ will be required.

\section{Acknowledgments}

We benefited in many ways from the daily contact with A. Blanco e N. Carolino. Some crucial equipment was kindly provided by F. Fraga.

This work was supported by the EU FP6 program via the contract RII3-CT-2003-506078 and by FCT and FEDER under project POCI/FP/63411/2005.

\section{REFERENCES}

1. P. Fonte, A. Smirnitski and M.C.S. Williams, Nucl. Instr. Meth., A 443(2000)201.

2. A. Akindinov et al., Nuclear Instr. and Meth. A490 (2002) 58-70.

3. P. Senger, The CBM experiment at FAIR, GSI Scientific Report 2004 (http://www.gsi.de/documents/QCD_CBMreport-2005-001.html).

4. AL-940CD charge-dissipative ceramics, Morgan Advanced Ceramics, 26 Madison Road, Fairfield, NJ 07004, USA.

5. A. Blanco et al., Nucl. Instr. and Meth A 535 (2004) $272-276$

6. G. Carboni, et al, Nucl. Instr. And Meth. A 498 (2003) 135.

7. L. Lopes, R.Ferreira Marques, P. Fonte, A.Pereira, V.Peskov, A.Policarpo, Nucl. Instr. and Meth. A533 (2004) 69-73.

8. C. Gustavino et al., Nucl. Instr. and Meth. A 527 (2004) 471.

9. D. González-Díaz et al., Nucl. Instr. and Meth.A 555 (2005) 72-79.

10. A. Akindinov, V. Golovin, A. Martemiyanov, V. Petrov, V. Plotnikov, A. Smirnitsky, K. Voloshin, 100 ps time-of-flight resolution of Dielectric Resistive Plate Chamber, http://arxiv.org/abs/physics/0502111.

11. A. Blanco et al., Nucl. Instr. and Meth A485 (2002) 328-342.

12. Ch. Finck et al., Proceedings of the XXXIX International Winter Meeting on Nuclear Physics, Bormio, 2001.

13. L. Lopes, R.Ferreira Marques, P. Fonte, A.Pereira, Accurate timing of gamma photons with high-rate Resistive Plate Chambers, proceedingsof the "7th International Conference on Position Sensitive Detectors", 12 - 16 September 2005, Liverpool, UK, Nucl. Instr. and Meth, in press.

14. A. V. Akindinov et al., Nucl. Instr. and Meth.A533 (2004) 93-97.

15. A. Blanco et al., Nucl. Instr. and Meth A508 (2003) 70-74. 